\section{hommes}

\section{Hommes \& migrations}

Revue française de référence sur les dynamiques

migratoires

1312 | 2015

Diasporas iraniennes

\title{
Le Raï fête ses 30 ans
}

\section{François Bensignor}

\section{Journals}

\section{Édition électronique}

URL : http://journals.openedition.org/hommesmigrations/3536

DOI : 10.4000/hommesmigrations.3536

ISSN : 2262-3353

\section{Éditeur}

Musée national de l'histoire de l'immigration

\section{Édition imprimée}

Date de publication : 1 octobre 2015

Pagination : 150-155

ISBN : 978-2-919040-33-9

ISSN : $1142-852 X$

\section{Référence électronique}

François Bensignor, "Le Raï fête ses 30 ans », Hommes \& migrations [En ligne], 1312 | 2015, mis en ligne le 01 juin 2016, consulté le 14 septembre 2020. URL : http://journals.openedition.org/ hommesmigrations/3536 


\title{
MUSIQUES
}

\section{LE RAÏ FÊTE SES 30 ANS}

\author{
par FRANÇOIS BENSIGNOR
}

A pparu sur la scène française en janvier 1986, le raï figure parmi les grands succès à porter à l'actif de la "génération Black Blanc Beur". La joie qui émanait de cette musique portée par une jeunesse assoiffée de liberté suscite un rien de nostalgie. Fêter les 30 ans du raï, c'est retrouver cet enthousiasme communicatif qui incitait au partage et au bien vivre ensemble. C'est du moins ce que souhaitaient les organisateurs du grand concert programmé au Zénith de Paris le vendredi 29 janvier 2016. Pour l'occasion, Universal Music publie un coffret de 5 CDs : Les 30 ans du raï. Chacun des trois premiers disques retrace une décennie de création. Le quatrième est consacré aux femmes du raï et le cinquième au raï en devenir.

Les péripéties traversées par le monde du raï et ses acteurs résonnent étrangement dans les temps troublés auxquels la société française est confrontée. Les survoler avec le recul peut être riche d'enseignements propices à la réflexion.

- 1982 - "Le raï entre par effraction à la télévision [algérienne] à travers un radio-crochet auquel participe Cheb Mami, écrit Rabah Mezouane*. Il chante El Marsam (Le refuge) sous les vivats d'une foule déchaînée. Quant au roi Khaled, il est de plus en plus courtisé par les 'libéraux' et on l'invite à chanter devant des hauts gradés en lui promettant de le dispenser de service militaire. Khaled, qui ne s'en laisse pas conter, réplique par Koubou Koubou (Remplissez les verres, repris sur l'album N'ssi N'ssi)."

- 1983 - À Oran, Chaba Zahouania publie sa première cassette de raï à 23 ans, un duo avec Cheb Hamid. Trois ans avant était sorti son tout premier enregistrement dans le style traditionnel féminin des meddahates. Elle enregistrera en duo avec tous les Cheb oranais du raï, excepté Sahraoui, et épousera le célèbre Boualem, principal producteur de raï à Oran avec ses éditions Disco Maghreb.

- 1984 - En Algérie, le président Chadli Bendjedid impose le code de la famille, qui revient à considérer la femme comme une mineure.

- 1985 - Première édition du Festival de raï d'Oran. Ce grand événement fédérateur du genre ne survivra pas à l'élection du Front islamique du salut (FIS) à la mairie de la ville cing ans plus tard, mais renaîtra en 1992.

- Alors que la cassette Hada Raykoum de Cheb Khaled, 25 ans, est vendue à 3 millions d'exemplaires, le pouvoir algérien, déclare le raï "partie intégrante du patrimoine national". "Le parcours de l'artiste de raï commençait dans les cabarets, à Oran, explique Michel Levy. C'est là que les producteurs venaient repérer les artistes. Tous les grands artistes de rai étaient sous contrat avec des maisons de production algériennes comme Disco Maghreb à Oran. Leurs enregistrements étaient proposés en licence à des détaillants. Un artiste se devait d'obtenir la reconnaissance du public qui achetait ses cassettes à Barbès, sous peine de voir ses productions disparaître des magasins. Avoir une cassette sur le marché signifiait que l'artiste pouvait animer les mariages, principale source de revenus à l'époque." - À Paris, Martin Meissonnier s'entiche d'une cassette de Cheb Khaled réalisée par les frères Rachid et Fethi Baba-Ahmed et se rend dans leur studio à Tlemcen. Il y rencontre Cheb Sahraoui, qui 
led, Cheb Sahraoui et Chaba Fadela, Cheb Hamid et Cheb Mami. Le festival reçoit le soutien du ministre de la Culture, Jack Lang, de l'Institut du monde arabe et de la Maison des cultures du monde. Les concerts sont présentés par Alain Maneval, journaliste musical à Europe 1, et Radio France est partenaire de l'événement.

C'est la première fois que Cheb Khaled se produit sur scène en France. Martin Meissonnier se souvient du concert : "Les 2000 places ont été prises d'assaut et les 2000 personnes restées dehors essayaient de se frayer un chemin à l'intérieur. Ce succès était tota-

Khaled - années 1980 (c) D.R.

le guide à Oran. "À l'époque, Khaled chantait dans les mariages toute la nuit, raconte Martin. I/ commençait pour les hommes et, à partir de 3 heures du matin, il changeait de répertoire et chantait pour les femmes. Il était assis en tailleur et s'accompagnait sur deux synthétiseurs : un Kawai 900 au son genre cornemuse, et un Crumar pour faire des nappes. Avec lui, Kouider au violon, une basse, une derbouka, un târ et une guitare. Des tentes étaient dressées sur les toits en terrasse et je me souviens d'une joute vocale démentielle entre Khaled et Sahraoui. Tous deux étaient alors les plus belles voix d'Oran."

- Michel Levy publie en France les deux premiers 33 tours de raï : "J'ai intitulé celui de Cheb Khaled Le roi du raï et celui de Cheb Mami Le prince du Raï, dit-il. J'ai entrepris d'en faire la promotion comme pour Daniel Guichard ou Charles Aznavour. Une programmatrice me dit: 'Mais Michel, ils chantent en arabe !...'Sur France Inter, une animatrice diffuse un titre dans son émission. Aussitôt, le standard de la radio est saturé d'appels racistes. Elle prend alors le contrepied : À la demande générale, voici un deuxième morceau !"'

- 1986 - 23 au 26 janvier : À l'initiative de Martin Meissonnier, la MC93 de Bobigny organise le Festival Raï. Au programme : Raïna Raï, Cheb Kha- lement inattendu pour un Khaled qui n'avait pas de passeport et dont le concert en banlieue n'avait bénéficié que d'une petite campagne de publicité. Mais il était déjà légendaire et sa légende l'avait précédé."

- 17 février : À Paris, la Grande Halle de La Villette accueille les pionniers de l'histoire vivante du raï, Cheikha Remitti, les Meddahates, Belkacem Bouteldja, Bellemou Messaoud, avec deux figures de la jeune génération, Djillali Amarna, le chanteur de Raina Raï, et Cheb Khaled.

- Le public de ces concerts historiques est constitué essentiellement de Maghrébins vivant en France.

- 1987 - À Oran, quelques islamistes exhortent les vendeurs de cassettes à baisser le volume de leur sono qui diffuse en boucle Baraka. Les paroles de ce tube sulfureux chanté en duo par Chaba Zahouania et Cheb Hasni disent : "Moi je l'ai prise et que les autres aillent se faire foutre / Hé, viens ce soir on n'en finira pas / Ce soir sa nuit se fera chez moi / Foutez-moi la paix, moi je n'en peux plus" (traduction de Bouziane Daoudi.)

- Les artistes oranais rentrés chez eux, le jeune Cheb Kader est le seul artiste de raï disponible en France. "Avec lui, nous avons fait 42 émissions de télévision en deux ans, explique Michel Levy, qui le produit. II a joué dans tous les concerts organisés par SOS Racisme et a connu un grand succès en 


\section{MUSIQUES}

Allemagne. L'hebdomadaire Der Spiegel lui avait consacré un article, qui en a entraîné d'autres dans toute la presse allemande. À 19 ans, Kader a joué une semaine à guichets fermés au Tempodrom de Berlin."

- 1988 - "Le raï et la chanson kabyle se font plus incisifs lors des émeutes de 1988, dans une Algérie où les animateurs de l'unique chaîne de télé et de Chaîne 1 (la radio en langue arabe) gardent toujours l'accent égyptien et 'oublient' sur leurs playlists la musique algérienne, écrit Rabah Mezouane*.

Cheb Mami, $1985 \oplus \mathrm{D} . \mathrm{R}$ Un titre de Khaled, El Harba Win (Fuir, mais où ?), adapté d'une composition de Idir, va servir de bande-son pour les manifestants lassés par le chadlisme corrompu." Ses paroles sont signées Mohamed Angar : "Où est passée la jeunesse ? / Où sont les gens courageux? / Les gros se goinfrent, / Les pauvres triment, / Les charlatans islamistes / Montrent leur vrai visage. / Alors quelle solution ?..." (traduction Rabah Mezouane).

- En Algérie, le lieutenant-colonel Hocine Snoussi, qui dirigeait l'office Riadh el Feth, organisme fondé sous la présidence de Chadli pour la consommation de luxe et une certaine culture permissive, est chargé de la production d'un album de Cheb Khaled. II la délègue en France au producteur Charles Talar et à l'auteur-compositeur Didier Barbelivien. Le jazzman algérien Safy Boutella, à qui sont confiés la direction musicale et les arrangements, fait appel à Martin Meissonnier pour la production artistique. Kutché, album splendide, va révéler le "roi du raï" au public international.

- 1989 - Venu vivre à Paris après deux ans de service militaire en Algérie, Cheb Mami raconte : "Les premiers jours à la caserne, à devoir marcher au pas, subir l'entraînement et tout ça, j'avais le moral à zéro. Je me demandais vraiment ce que faisais là. Et puis, au bout d'une quinzaine de jours, même le général était au courant que j'étais dans la

caserne. II m'a invité à venir dans son bureau, où j'ai été bien accueilli. (...) J'ai pu faire une tournée dans une vingtaine de casernes et enregistrer deux cassettes. À la fin de mon incorporation, j'ai fait les deux plus beaux concerts de ma carrière à Alger: 40000 spectateurs à chaque concert !... C'était pendant le ramadan, après les événements de l'automne 1988. Il y avait des familles, beaucoup de jeunes. Après mon séjour et mes enregistrements en France, mon nom avait pris de la valeur en Algérie. Mais je ne réalisais pas à quel point... J'avais un trac fou !"

- 1990 - 21 janvier : Cheb Khaled triomphe au Bataclan.

- 21 juin : En Algérie, le FIS remporte les élections municipales avec $54,3 \%$ des suffrages. À Oran, la nouvelle équipe islamiste s'empresse d'interdire toute manifestation raï. Les Cheb et les Chaba sont sur liste noire.

- 1991 - 14 juillet : À l'occasion de la fête nationale, le gouvernement français initie à New York un grand concert à Central Park. En têtes d'affiche : Khaled, le roi du raï, Mory Kante, le griot électrique, les Gipsy Kings, les Gitans de Camargue.

- 26 décembre : En Algérie, le FIS remporte les élections législatives avec $47,3 \%$ des voix. 
- 1992 - Contraint à la démission le 11 janvier, le président Chadli laisse la place à Mohamed Bouiaf, leader historique du Front de libération nationale (FLN) en exil au Maroc depuis trente ans. Celuici annule le second tour des élections, proclame l'état d'urgence, fait interner des milliers d'islamistes et dissoudre le FIS. II sera assassiné le 29 juin à Annaba.

- Bouziane Daoudi recueille le témoignage de Cheb Hasni pour Libération:"Avec le FIS, ni concert, ni festival. Oran mourait. Aujourd'hui, ils lui ont coupé le souffle, le FIS ne respire même plus. Les choses revivent. (...) Ils (les barbus) viennent souvent chez moi : 'Tu as une belle voix, pourquoi ne viendrais-tu pas nous faire le muezzin ? Les gens t'adorent, s'ils te voient prier, ils viendront prier, eux aussi.'Ce ne sont pas les barbes sévères qui viennent, ce sont les jeunes modernes. Souvent, on connait le mec: un voisin du quartier qui buvait avant de devenir 'frère'. II rigole, parle de tout, n'a pas le discours ringard. II sait parler." Sa chanson Le visa est alors sur toutes les lèvres : "J'avais décidé de rejoindre ma bienaimée / Honte à vous, vous m'avez peiné / Vous avez été jusqu'à me priver du visa / Vous voulez ma mort ou quoi? / Je vais me saouler et tout casser / Pourquoi cette injustice / Alors que mon passeport est valide / Et que je ne tiens pas à faire d'histoires..." (traduction Rabah Mezouane).

- Pour la première fois, Chaba Zahouania révèle son visage dans une vidéo. Jusqu'alors, les pochettes de ses cassettes présentaient les photos de belles blondes aux yeux bleus... Ce qui avait permis à une autre chanteuse d'usurper son nom pour démarcher des concerts.

- En France, Khaled rejoint l'écurie Barclay. Didi, chanson extraite de son album paru en février, s'installe pendant vingt semaines au Top 50. Traduit en grec, en espagnol, en hébreu, etc., le titre est plébiscité en Inde et en Indonésie. Débarrassé du qualificatif "cheb" (jeune), Khaled séduit le monde anglo-saxon. Ses productions s'orientent vers un public de plus en plus large. En novembre, il triomphe au Bataclan pendant une semaine.

- 1993 - Khaled déclare à Bertrand Tessier pour Télécable: "Même si on me déroulait le tapis rouge, je n'irais pas en Algérie. On ne peut pas chanter pendant qu'il y a des gens qui meurent pour des raisons politiques. Je ne veux pas risquer ma vie. En ce moment, ils assassinent des médecins, des intellectuels, des journalistes, des universitaires. Ils veulent conduire le pays à la guerre civile. II parait que je suis sur la liste..."
Cheb Hasni - 1992-94 (c) D.R.

- 23 décembre : L'Armée islamique du salut (AIS) enjoint les disquaires algériens de cesser la vente de disques ou de cassettes sous peine de mort.

- 1994 - 29 septembre : Cheb Hasni, adulé dans le monde arabe avec son “raï love", est abattu à Oran. 


\section{MUSIQUES}

-1995-15 février : Rachid Baba-Ahmed, célèbre producteur et compositeur de raï installé à Tlemcen, est assassiné à Oran pendant le ramadan.

Les rares vedettes du raï qui habitent encore en Algérie rejoignent la France, où vivent déjà Khaled, Mami, Fadela, Sahraoui, Zahouania, Tahar, Nasro et les autres.

- 3 et 4 mars : Cheb Mami remplit le Bataclan pour deux soirées.
- 1996 - Khaled séduit le grand public français avec Aïcha, un tube écrit pour lui par Jean-Jacques Goldman, qui fera l'objet de multiples versions à l'international : Afrique, Espagne, Turquie, Grèce, Israël, etc.

- 1997 - À 19 ans, Faudel, né et grandi à Mantesla-Jolie, accède au Top 50 avec son tube Tellement N'Brick (Tellement je t'aime). Son premier album, Baïda, sera vendu à près de 300000 exemplaires.

- 1998 - 26 septembre : À Paris, plus de 17000 spectateurs affluent au Palais omnisport de Bercy pour 1, 2, 3, Soleil. Le concert événement qui reprend le titre du film de Bertrand Blier, dont Khaled a composé la musique en 1993, réunit Khaled, Rachid Taha et le jeune Faudel avec un orchestre de 53 musiciens.

- "Paradoxalement, on voit le chant du cygne dans 1, 2, 3, Soleil, analyse Rabah Mezouane. On est en septembre 1998, peu après la victoire de l'équipe de France de football en Coupe du Monde. Le 'Black Blanc Beur'est glorifié. Mais malgré les ventes record de l'album, qui s'écoule à près d'un million d'exemplaires, on va constater le reflux du rai."

- 1999 - Faudel reçoit le titre de "Révélation de l'année" aux Victoires de la musique.

- 2000 - Nouar, l'album de Cheikha Rimitti arrangé par Mohammed Maghni, as des claviers funky à la mode algérienne, est élu Grand Prix de l'Académie Charles Cros. Une consécration pour la "mère spirituelle" du raii. Les Cheb se sont beaucoup inspirés de la créativité prolixe de la chanteuse installée à Paris dès 1979. Sa place de doyenne sentencieuse au grain de voix impressionnant n'a jamais été contestée. 
Khaled - années $1980 \odot$ D.R.

- 2001 - 11 septembre : Les attentats de New York et de Washington mettent un coup d'arrêt terrible à la diffusion du raï dans les pays occidentaux. Dès lors, les artistes rencontrent d'énormes difficultés à tourner et à faire diffuser leurs œuvres sur les radios de grande écoute.

- 2003 - Cheb Mami enregistre en duo avec Sting la chanson Desert Rose, qui deviendra un tube.

- 2004 - L'album et la vidéo du concert de Cheb Mami au Grand Rex conforte son statut d'artiste majeur.

- Premier album du collectif Raïn'B Fever initié par DJ Kore \& Scalp. S'y retrouvent mixés les jeunes pointures du raï, du R'n'B et du Hip-Hop oriental. Cette nouvelle tendance ne cessera de s'affirmer jusqu'en 2011 avec quatre compilations successives.

- 2005 - Le raï oranais est mis à l'honneur au festival Les Escales de Saint-Nazaire, avec Cheba Djamila et le groupe Liberté. Elle témoigne : "Aujourd'hui, les cabarets se sont multipliés et les chanteurs sont si nombreux qu'on ne gagne plus grand-chose dans les cabarets (...). Rien ne s'est arrêté après la mort de Hasni. Bien sûr, il y a eu la peur, mais la musique a continué et le raï est là comme avant."
- 2006 - 15 mai : Cheikha Rimitti, qui vient de fêter ses 83 ans, est emportée par une crise cardiaque. Deux jours plus tôt, elle chantait sur la scène du Zénith, entourée de la jeune génération, Khaled en tête.

-2007-19 au 22 juillet : Première édition du Festival international du raï à Oujda, Maroc. Oujda confirme sa proximité géographique et culturelle avec Oran et sa région. Depuis huit ans, le festival a défendu sa place centrale dans la promotion du raï, tout en s'ouvrant à une grande diversité de genres musicaux. En 2015, ses organisateurs ont initié une démarche d'inscription de la culture raï au patrimoine mondial de l'Unesco.

- 2009 - 3 juillet : Cheb Mami est condamné à cing ans de prison ferme pour tentative d'avortement forcé sur son ex-compagne. II obtiendra une liberté conditionnelle le 23 mars 2011.

- Khaled enregistre avec Magic System le tube Même pasfatigué,réalisé parDJ Korede Raï'n'B Fever. - Reda Taliani enregistre Partir loin avec les rappeurs humoristiques du 113. La vidéo du titre totalise aujourd'hui plus de 9,5 millions de vues sur YouTube.

- Années 2010 - "Ce qui marque le raï dans les années 2010, c'est l'effet Raï n'B, explique Rabah Mezouane. En s'alliant avec le hip-hop, le raï trouve une nouvelle voie, qui dessine l'orientation vers un genre musical nouveau, où les styles et les artistes se mélangent."

- Coffret de 5 CDs, Les 30 ans du Raï (Universal Music), sorti le 15 janvier 2016.

* Télérama, numéro hors série, mars 1995 : Algérie, la culture face à la terreur. 